\title{
O ESTADO DA CIÊNCIA DO DESENVOLVIMENTO DE BIOSSENSORES A NÍVEL NACIONAL
}

\author{
A. M. SALGADO ${ }^{1}$, G. JACOBY ${ }^{2}$ e L. A. de PAULA ${ }^{3}$ \\ ${ }^{1}$ Universidade Federal do Rio de Janeiro, Escola de Química, Departamento de Engenharia \\ Bioquímica, Laboratório de Sensores Biológicos \\ ${ }^{2 e} 3$ Universidade Federal do Rio de Janeiro, Escola de Química, Laboratório de Sensores \\ Biológicos \\ E-mail para contato: andrea@eq.ufrj.br
}

\begin{abstract}
RESUMO - Biossensores vêm surgindo como uma alternativa a métodos clássicos de qualificação e quantificação de analitos, por sua alta especificidade, baixo tempo de resposta, potencialidade de baixo custo e portabilidade. Desse modo, no presente trabalho, buscou-se avaliar o estado da ciência do desenvolvimento de biossensores a nível nacional, pesquisando-se, para isso, patentes depositadas no período de 1993 a 2012 e artigos publicados por autores brasileiros no período de 2002 a 2012, sendo separados de acordo com: tipo de componente biológico, tipo de transdutor, ano de publicação do artigo ou de depósito da patente e instituição e estado brasileiro responsável por fazê-lo. Observou-se a tendência de aumento de estudos em biossensores a nível nacional, em que regiões isso mais tem ocorrido, os principais parâmetros escolhidos para a confecção do biossensor e quais estudos sobre biossensores geraram dispositivos com perspectivas reais de comercialização.
\end{abstract}

\section{INTRODUÇÃO}

$\mathrm{O}$ advento de novas tecnologias em controle e automação e o rápido progresso na miniaturização de componentes eletrônicos estende-se hoje entre os mais diversos setores da ciência. Sua abrangência passou a incluir não só áreas tradicionais, mas também campos antes inimagináveis, tais como cosméticos, alimentos, saúde e ambiental.

Ao mesmo tempo, a biotecnologia desponta como o grande potencial de sustentabilidade, capaz de complementar ou até mesmo substituir processos tradicionais químicos, muitas vezes caros, menos eficientes e agressivos ao meio ambiente. A biotecnologia abriu horizonte para o desenvolvimento e aplicação de novos dispositivos que pretendem ser melhores que os tradicionais ou, complementares a eles, melhorando seu desempenho quanto a rapidez, especificidade e custos.

É justamente nesse contexto, da pesquisa em biotecnologia e bioengenharia, que o tema de biossensores se insere. Combinando a seletividade e a sensibilidade da biologia ao potencial de processamento dos componentes microeletrônicos, os biossensores vêm surgindo como alternativa de detecção de analitos (Cavalcanti, 2010). Sua proposta é funcionar como uma ferramenta analítica capaz de substituir ou complementar os métodos tradicionais já consagrados. 
Oficialmente, segundo a IUPAC (International Union of Pure and Applied Chemistry), um biossensor pode ser definido como "um instrumento integrado que é capaz de fornecer uma informação analítica específica quantitativa ou semi-quantitativa através do uso de um elemento de reconhecimento biológico que está em contato direto com o elemento transdutor.

Biossensores acoplam a funcionalidade de um elemento biológico, altamente específico e seletivo, ao poder de sensibilidade de ferramentas eletrônicas capazes de captarem o sinal gerado e o transmitirem como um sinal mensurável. Estes possibilitam ainda a obtenção de medidas de monitoramento em campo on line e in situ, o que fornece resultados muitos mais rápidos e ainda podem fornecer informações acerca da real biodisponibilidade ou biotoxicidade de uma substância em determinada biota, sendo uma vantagem para monitoramento de locais contaminados (Patacas, 2007).

Por ser um instrumento de análise que envolve a transdução do produto da atividade metabólica em sinal quantificável e processável, o componente biológico (enzimas, cofatores, receptores, anticorpos, células de micro-organismos, tecidos de plantas e animais ou organelas) é de fundamental importância para o biossensor, pois confere a especificidade da análise (Salgado, 2001).

No contexto dos biossensores, o transdutor é o elemento responsável por transformar o produto da reação biológica em um sinal elétrico que possa ser quantificado e interpretado (Malhotra, 2005). Os elementos de transdução mais usados na confecção dos biossensores são: eletroquímicos (potenciométricos, amperométricos), ópticos, calorimétricos e piezoelétricos.

Sendo ainda um campo essencialmente teórico de estudos, os biossensores têm apresentando muitas inovações em escala de bancada. Tais melhorias visam à amenização de pendências que impedem que se alcance a proposta inicial de um dispositivo barato, simples e portátil, sejam elas associadas ao elemento biológico utilizado ou ao mecanismo transdutor. Desse modo, faz-se necessário um pequeno levantamento acerca das últimas tendências a fím de melhorar a performance dos biossensores propostos (Mello e Kubota, 2002).

Atualmente, já existem no Brasil alguns biossensores patenteados e até mesmo comercializados, porém a maior parte dos trabalhos ainda está em nível de pesquisa. Assim, mostra-se necessário um profundo levantamento e estudo acerca do estado da ciência do desenvolvimento desses sensores a nível nacional. Nesse sentido, o presente trabalho propõe-se a buscar dados em artigos e patentes e tratá-los estatisticamente a fim de se obterem respostas acerca do foco que se vem dando ao desenvolvimento dos biossensores no Brasil.

\section{METODOLOGIA DE PESQUISA}

\section{1-Patentes}

Com o intuito de avaliar a produção científica e tecnológica no Brasil nos últimos anos na área de biossensores, foi feito um levantamento de todas as patentes que já foram depositadas, sejam de autores brasileiros ou não, no país. Para tal, usou-se como base de dados o portal do INPI (Instituto Nacional da Propriedade Industrial). Assim, foi realizada uma pesquisa no endereço eletrônico http://www.inpi.gov.br., abrangendo o período de 1993 a 2012. 
Em acordo com o objetivo do trabalho, foi feita um busca de possíveis sinônimos para o termo biossensor, cujos resultados foram usados na pesquisa. Foi inserida na busca como palavra chave o termo "biossensor", além desta, outros termos como "Biossensores", "Sensor Biológico", "Sensores Biológicos", "Biosensor" e "Biosensores" foram também usados.

\section{2- Artigos}

A fim de buscar informações qualitativas e quantitativas dos artigos gerados por brasileiros com o tema biossensores, foi feita uma busca no endereço eletrônico da base de periódicos Portal Capes (http://www.periodicos.capes.gov.br/) utilizando a ferramenta de Busca Avançada do site.

Como parte do intuito do presente estudo é a realização de uma análise quantitativa dos artigos pesquisados, procurou-se não refinar muito os resultados para que não houvesse perda de informações relevantes. Desse modo, foram pesquisadas as seguintes palavras-chave: biosensor, biossensor, biosensores, biossensores, sensor biológico, sensores biológicos, sensor. A Data de Publicação foi definida para incluir resultados dos últimos dez anos e definiu-se o Tipo de material como Artigos.

\section{3- Tratamento Estatístico dos Dados}

No caso dos artigos, todas as citações encontradas foram percorridas e aquelas de autores brasileiros foram selecionadas. No caso das patentes, foram pesquisadas não só aquelas depositadas no Brasil por autores brasileiros, mas também patentes estrangeiras com depósito internacional via PCT - Tratado de Cooperação de Patentes. Em cada um dos artigos ou patentes correspondentes às citações selecionadas, buscaram-se as seguintes informações: título, ano (para patentes ano de pedido de depósito), tipo de componente biológico, transdutor, área de aplicação, instituição responsável (no caso de patentes foi verificado os tipos de organizações depositantes, Instituições de Ensino e Institutos Públicos de Pesquisa (IEIPP) ou Empresas e Institutos Privados de Pesquisa (EIPP)), e estado. Esses dados foram, armazenados em uma planilha no programa Excel Versão 2007, e as informações trabalhadas de maneira quantitativa.

A fim de trabalhar estatisticamente os dados obtidos, foram utilizados métodos gráficos, facilitando, assim, a busca por padrões e relações, a confirmação de expectativas sobre os dados e a apresentação de resultados. Nesse sentido, cada um dos parâmetros relacionados aos biossensores foi tratado como uma variável qualitativa, de modo que as distribuições de suas frequências com relação à amostragem total puderam ser calculadas e expressadas graficamente como resultados (Bussab e Morettin, 2011).

\section{RESULTADOS E DISCUSSÕES}

\section{1- Patentes}

Como o Portal do INPI não possibilita a busca por país de origem da patente, e levando em conta que o resultado obtido revelou que a grande maioria das patentes depositadas atualmente no país nesse assunto foram desenvolvidas em outros países, optou-se por não descartar as patentes com depósito internacional via PCT - Tratado de Cooperação de Patentes. O PCT é um tratado multilateral que permite requerer a proteção patentária de uma invenção, simultaneamente, num grande número de países, por intermédio do depósito de um único Pedido Internacional de patente. 
A produção de patentes relacionadas a sensores biológicos passou por flutuações entre os anos de 1993 e 2012, como mostra o gráfico da Figura 1a. Na década de 90, tivemos um total de 9 patentes depositadas. Somando-se todas as patentes depositadas entre os anos de 2000 e 2011, observamos o expressivo aumento de $500 \%$ na produção, chegando a uma soma total de 55 no início de 2011, como mostra a Figura 1b. No ano de 2012, até a data da pesquisa, não havia patentes depositadas nem pedidos de depósito sendo avaliados.

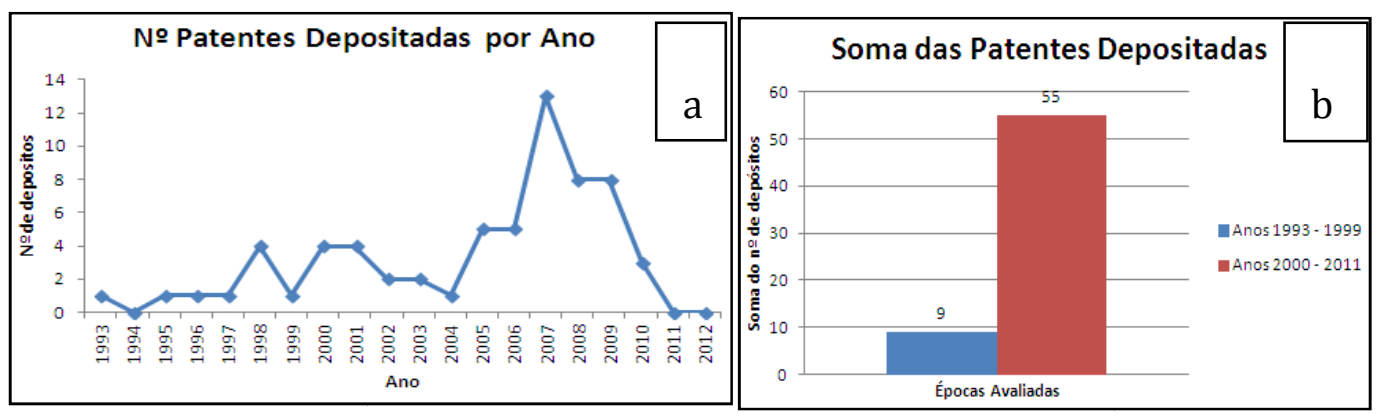

Figura 1-(a) Depósito de patentes em Biossensores nos últimos 20 anos e (b)Soma do número de patentes depositadas entre 1993 e 1999 e entre 2000 e 2011.

O gráfico representado na Figura 2a traz uma análise demográfica da produção de patentes em biossensores, destacando, os Estados Unidos, que lideram o ranking em produção com 32 patentes. Em seguida, temos empatados a Organização Européia de Patentes e o Brasil, com destaque para os estados do Rio de Janeiro e São Paulo, sendo este último responsável por $90 \%$ do total já produzido no Brasil. A Figura $2 \mathrm{~b}$ mostra as organizações responsáveis pelas patentes, cerca de $70 \%$ são de autoria de EIPP, $20 \%$ de IEIPP. No Brasil, das 10 patentes que foram encontradas, 9 são oriundas de IEPP, destacando a Universidade Estadual de Campinas UNICAMP (SP) como principal depositante do país em se tratando de biossensores.

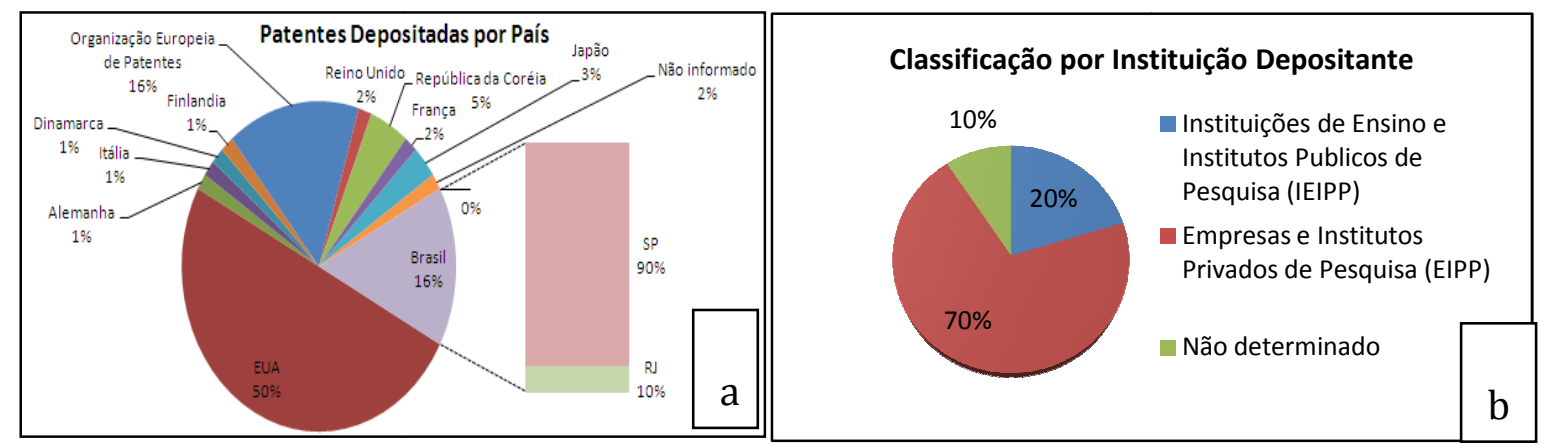

Figura 2-(a)- Análise demográfica da produção de patentes em biossensores e (b) organizações responsáveis pelas patentes.

Os gráficos mostrados nas Figuras 3(a) e 3 (b) a seguir representam o numero de patentes depositadas de acordo com os tipos de biocomponentes e transdutores usados nos biossensores. 


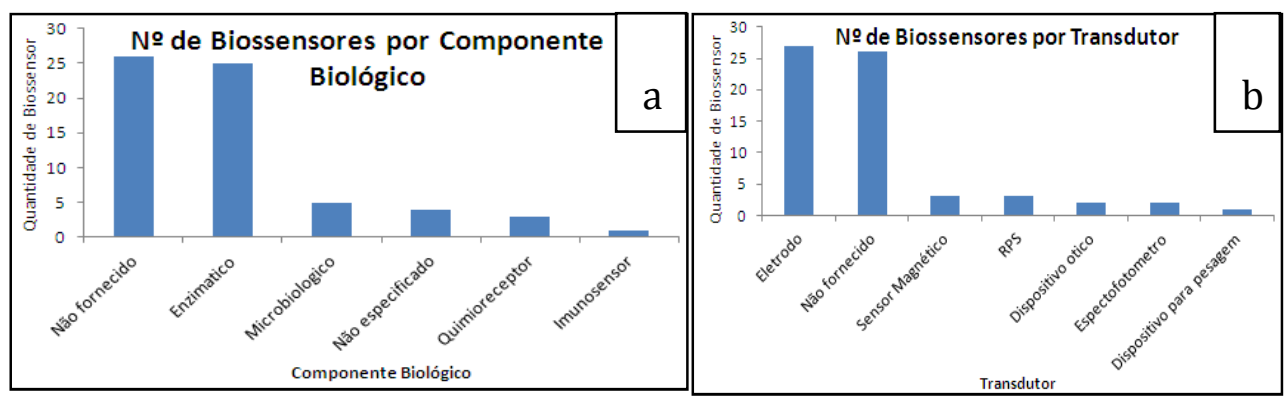

Figura 3(a) Número de biossensores por tipo de componente biológico e 3(b) de transdutor.

O biossensor enzimático (em especial para aplicação de medição de glicose no sangue) foi o que mais gerou patentes, seguido do microbiológico. No caso do sistema de transdução, temos em destaque o eletrodo, o que é explicado pois estes sistemas são baseados em enzimas redox (oxiredutases), e existe um grande número destas enzimas comercialmente disponíveis que podem atuar em ácidos graxos, açúcares, aminoácidos, aldeídos e fenóis.

\section{2-Artigos}

Foram encontrados um total de 144 artigos na área de biossensores. Inicialmente, os resultados foram processados a fim de se determinar em que áreas de aplicação está a maior concentração dos estudos em biossensores mostrado na Figura 4a e às regiões do Brasil de maior número de artigos sobre biossensores publicados mostrado na figura $4 \mathrm{~b}$.

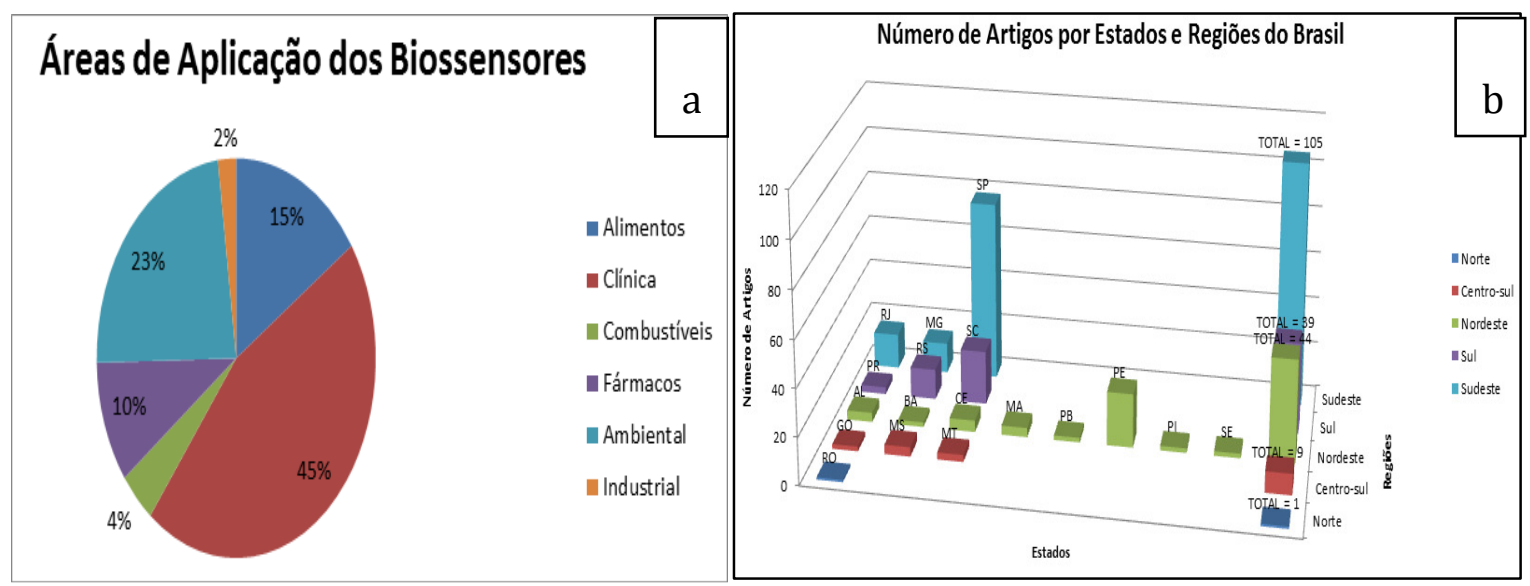

Figura 4-(a) Áreas de aplicação dos biossensores detectadas e (b) Número de artigos por estados e por regiões do Brasil

Em relação a área de aplicação nota-se que a maior parte dos biossensores estudados é voltada para a área clínica, $45 \%$ do total de artigos selecionados. A área clínica possui bastante potencial para exploração dos biossensores, uma vez que usualmente necessita de dispositivos de sensoriamento capazes de fornecer respostas rápidas e precisas. A segunda área de maior exploração dos instrumentos biossensores é a ambiental, $23 \%$ do total de artigos no período de estudo. Esse fato é interessante no sentido de que vem acompanhando uma tendência global de 
maior preocupação com o meio ambiente e os efeitos a longo prazo da presença de contaminantes no mesmo.

Em relação as regiões do Brasil, a produção de artigos da região Sudeste supera em muito a que ocorre em outras regiões brasileiras, sendo São Paulo o estado com maior número de artigos. A segunda região que mais tem publicado artigos sobre o assunto é o Nordeste. Apesar de poder parecer que o grande número de estados dessa região favorece a posição da mesma, nota-se que a maioria dos estados não possui grande contribuição científica; em verdade, o estado de Pernambuco desponta como grande produtor de conhecimentos científicos sobre biossensores transformados em artigos.

A produção nacional de artigos com o tema biossensores vem experimentando significativo aumento nos últimos 10 anos, conforme explicitado na Figura 5. Esse aumento pode ser justificado pela própria eclosão da biotecnologia como uma ciência aplicada o que ajudou na disseminação da pesquisa em torno de biossensores, pela descoberta de novas técnicas de obtenção de enzimas, o que permitiu que os biossensores enzimáticos, pudessem ter seu custo expressivamente diminuído, além de adquirirem maior estabilidade e tempo de vida, e pelo advento do estudo em biossensores e a preocupação com o meio ambiente a níveis globais, demandando instrumentos analíticos mais práticos e capazes de fornecerem medidas precisas e de biodisponibilidade.

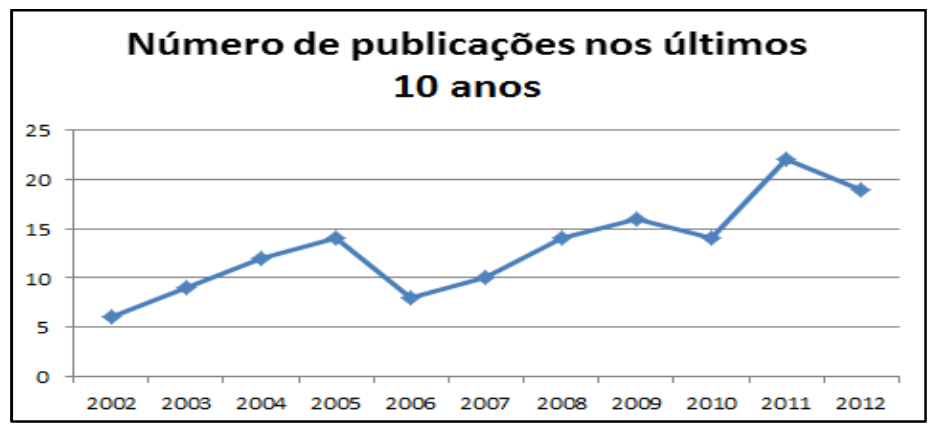

Figura 5- Número de artigos publicados sobre biossensores nos últimos 10 anos

É interessante ressaltar que a grande maioria dos biossensores desenvolvidos a nível de pesquisa por cientistas brasileiros utiliza enzimas como o componente biológico, como se observa na Figura 6a. Com relação ao tipo de transdutor mais utilizado nos biossensores referentes aos artigos pesquisados, observa-se que, em geral, os transdutores eletroquímicos são os predominantes como pode ser visto na figura $6 \mathrm{~b}$. Especificamente, transdutores amperométrico e potenciométrico têm sido os alvos de maior interesse de estudos, o que pode ser justificado pelo fato de esse tipo de transdução permitir instrumentação relativamente simples, além de bastante específica e, em vários casos, não influenciada pela presença de outras espécies químicas em solução. 

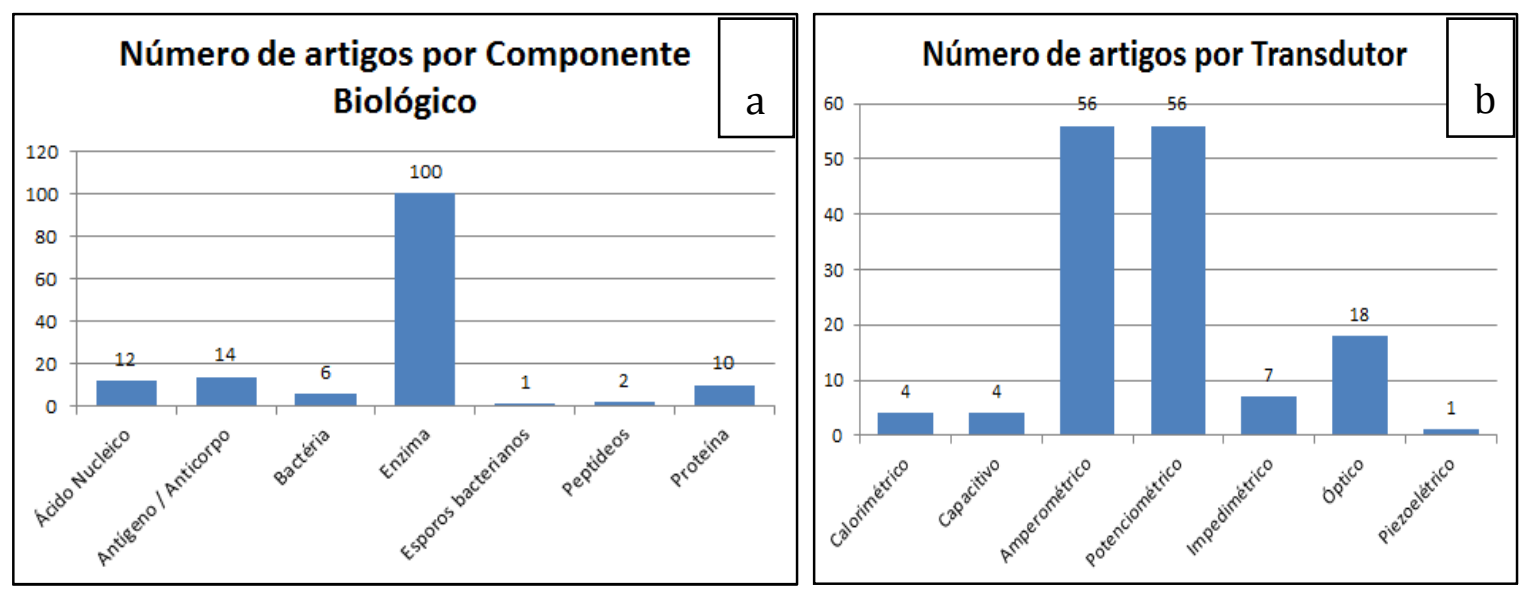

Figura 6(a) Número de artigos encontrados classificados pelo componente biológico utilizado e (b) por tipo de transdutor.

\section{3- Análise Geral}

Realizou-se uma análise entre as patentes caso a caso, comparando-as com os artigos publicados. Nesse sentido, foram comparados os seguintes parâmetros: o nome dos responsáveis pelo depósito da patente com relação ao nome dos autores de artigos; a data de depósito da patente com a data de publicação dos artigos; o conteúdo da patente depositada e dos artigos, com relação a itens como área de aplicação, tipo de transdutor, componente biológico e instituição e estado do Brasil em que a patente ou artigo foram desenvolvidos.

As patentes selecionadas nesta pesquisa incluem não apenas biossensores desenvolvidos no Brasil, mas também aquelas desenvolvidas em outros países por autores estrangeiros com pedido de depósito no Brasil. Por outro lado, os artigos pesquisados correspondem apenas a publicações desenvolvidas em instituições brasileiras. Desse modo, só foi possível a comparação entre patentes e artigos desenvolvidos no Brasil e por autores brasileiros, mais especificamente em universidades brasileiras, uma vez que nenhum dos artigos selecionados foi publicado por empresas privadas. Nesse sentido, verificou-se que alguns estudos publicados em artigos deram origem ao depósito de patentes, porém isso ocorreu apenas no estado de São Paulo, mais especificamente, nas universidades públicas USP, Unicamp e UNESP e na Fundação de Amparo à Pesquisa do Estado de São Paulo. Assim, considerando um total de 144 artigos brasileiros publicados entre os anos de 2002 a 2012, estima-se que apenas 9\% deles deu origem a depósitos de patentes.

\section{4- CONCLUSÕES}

A partir dos dados obtidos e do estudo realizado ao longo deste trabalho, observa-se uma tendência não só no Brasil como no mundo o fato de pesquisas sobre um tema começarem no meio acadêmico e, somente após um período onde se verifica a potencialidade do estudo em gerar um produto com aplicabilidade comercial, começam os investimentos da indústria e com isso, o aumento do numero de patentes. De acordo com os números, as IEIPP estão muito mais preocupados em gerar artigos. A aplicação comercial dos biossensores continua bastante restrita, visto o pequeno número de patentes brasileiras com esse tema depositadas nos últimos 10 anos. Essencialmente, trata-se ainda de uma área de pesquisa em escala de bancada, mas que apresenta potencial. 


\section{5- REFERÊNCIAS}

ARYA, S.K.; DATTA, M.; MALHOTRA, B.D. Recent a advances in cholesterol biosensor. Bios. and Bioelec., v. 23, p. 1083-1100, 2008.

BUSSAB, W.O.; MORETTIN, P.A. Estatística Básica. $7^{\text {a }}$ edição. Rio de Janeiro. Editora. Saraiva. Rio de Janeiro, 2011.

CAVALCANTI, G.S. Aplicação de biossensor bioluminescente na detecção do HPA naftaleno em solo contaminado com óleo cru. Dissertação Mestrado em Tec. Proc. Quim. e Bioq. Escola de Química. Universidade Federal do Rio de Janeiro. Rio de Janeiro, 2010.

MALHOTRA, B. D. Recent trends in biosensors. Current Apl. Phy., n. 5, p. 92-97, 2005.

MELLO, L.D.; KUBOTA, L.T. Review of the use of biosensors as analytical tools in the food and drink industries. Food Chem., v. 77, p. 237-256, 2002.

PATACAS, R.C. Desenvolvimento, caracterização e otimização de um biossensor amperométrico para a determinação de nitrato baseado em microinterfaces gelificadas. Dissertação (Mestrado em Ciências). Faculdade de Ciências. Universidade do Porto, Porto, 2007.

PORTAL DO INPI. Disponível em: $<$ http://www.inpi.gov.br/images/stories/downloads/informacao_tecnologica /pdf/Nanomateriais_2011_1sem_N60.pdf> Acesso em 01 de dezembro de 2013.

PORTAL CAPES. Disponível em: $<$ http://www.periodicos.capes.gov.br>. Acesso em outubro e novembro de 2013.

SALGADO, A.M. Desenvolvimento e aplicação de sensores e sistemas de monitoração de biomassa, etanol e de substrato por modelo. Tese de doutorado, em Tec. Proc. Quim. e Bioq.Escola de Química. Universidade Federal do Rio de Janeiro. Rio de Janeiro, 2001. 